\title{
Fault detection on bearings coupled to permanent magnet DC motors by using a generalized Takagi-Sugeno PI observer
}

\author{
C. Martínez-García ${ }^{1}$, G.-L. Osorio-Gordillo ${ }^{1}$, C.-M. Astorga-Zaragoza ${ }^{1}$ and V. Puig ${ }^{2}$
}

\begin{abstract}
This paper presents a fault detection system for rotative machinery. A permanent-magnet DC motor is used as case of study. The main idea is to estimate on-line the non-load torque $\left(T_{0}\right)$ in order to monitor the bearing health condition. The fault detection system is based on the design of a generalized Takagi-Sugeno PI (proportional-integral) observer. The main advantage of this approach is that it can be easily implemented because the observer gains are obtained by solving a set of LMIs (linear matrix inequalities). Moreover, the method can be extended to more complicated nonlinear systems by using the Takagi-Sugeno approach. A simulation is performed to show that this fault detection scheme can be applied to detect abrupt faults on rotative machinery which can lead the system to undesirable performance caused by vibrations or breakdown.

Index Terms-Proportional-integral observer, Takagi-Sugeno system, fault detection, LMIs (linear matrix inequalities).
\end{abstract}

\section{INTRODUCTION}

Rotative machines are used in almost all manufacturing processes performing a wide diversity of tasks. Examples of rotative machines frequently found in industries are pumps, motors, compressors and generators.

A common source of faults in this kind of machinery are the bearings whose main function is to prevent vibrations along the shaft or along the mechanical components coupled with the rotatory system of the machine.

When bearings are used as support, it is crucial to prevent overheating and fatigue of the manufacturing materials caused mainly by friction. Friction causes wear which in turn causes vibration on the whole rotatory system.

Friction effects on bearings can be minimized by selecting carefully the quality of the manufacturing materials, the quality of lubricants or by selecting adequately the design of the bearings (ball bearings, roller bearings, thrust bearing) depending on the application to be used.

Bearing breakdown can cause serious damage on the equipment and in consequence important economic losses. This is the main reason because bearings are the object of permanent maintenance programs in most industries and several works are devoted to propose methods to monitor the bearing health condition.

For instance in [1], the authors compute a mathematical model of the DC motor based on the block-pulse function series method [2] and then a multilayer neural network

\footnotetext{
${ }^{1}$ C. Martínez-García, G.-L. Osorio-Gordillo and C.-M. Astorga-Zaragoza are with the Centro Nacional de Investigación y Desarrollo Tecnológico. Interior Internado Palmira s/n, Col. Palmira Cuernavaca, Morelos, México. mgci@cenidet. edu. $\mathrm{mx}$

${ }^{2}$ V. Puig is with the Universitat Politécnica de Catalunya. C. Jordi Girona, 31, 08034 Barcelona, Spain.
}

configuration to detect changes on the model parameters. Neural networks combined with other techniques such as fuzzy logic have also been used for fault diagnosis of DC motors [3]. Another recent work [4] uses a fault detection method based on a high-gain observer [5] to detect changes on the nominal parameters of a DC motor. However, the use of complex Algebraic Geometric techniques represent a limitation to extend this method to other rotative machines having more complex mathematical nonlinear models.

Because a variation on the value of the load torque $\left(T_{0}\right)$ under unchanged operating conditions can be associated with a problem on the bearings, in this work we propose a method to supervise the friction coefficient through the online estimation of $T_{0}$. The fault detection system is based on a generalized PI (proportional-integral) observer (a more general case of this observer was developed for descriptor systems in [6]) which is designed by using a Takagi-Sugeno model of the DC motor presented in Section II. The structure of this generalized PI observer has been widely studied in recent works ([6], [7], [8], [9], [10]). It is based on the dynamic observers presented in [11] and [12]. The generalized PI observer structure presents an alternative state estimation which can be considered as more general than the Luenberger proportional-integral and proportional observers.

The main advantage of this observer is that it only requires a practical computation of the gains by solving a set of LMIs (linear matrix inequalities). Another important feature of the observer is that it can be extended to other processes having the general Takagi-Sugeno representation given in Section III. In Section IV, a simulation is performed in order to evaluate the fault detection capabilities of the proposed scheme. Finally, in Section V some general conclusions are given.

\section{MODEL DESCRIPTION}

\section{A. System under study}

Consider a typical mathematical model of a permanentmagnet DC motor [1]:

$$
\begin{aligned}
\dot{i}_{a}(t) & =-\frac{R_{a}}{L} i_{a}(t)-\frac{K_{e}}{L} V_{m}(t)+\frac{1}{L} u(t) \\
\dot{V}_{m}(t) & =\frac{K_{T}}{J_{1}} i_{a}(t)-\left(\frac{f_{r}+f_{p}}{J_{1}} V_{m}(t)\right) V_{m}(t)-\frac{T_{0}(t)-T_{2}}{J_{1}}
\end{aligned}
$$

where the states are the armature current $i_{a}(t)$ and the normalized rotational speed $V_{m}(t), u(t)$ is the input voltage. 
$T_{0}$ and $T_{2}$ are the non-load and load torque respectively. The DC motor parameters are shown in Table I.

TABLE I: Variables involved in the nonlinear model of a DC motor

\begin{tabular}{|c|l|c|}
\hline Variable & Description & Units \\
\hline \hline$R_{a}$ & Armature resistance & $\Omega$ \\
\hline$L$ & Inductance & $H$ \\
\hline$K_{e}$ & Back-emf coefficient & $V / r p m$ \\
\hline$K_{T}$ & Torque-current coefficient & $N \cdot m / A$ \\
\hline$J_{1}$ & $\begin{array}{l}\text { Normalized inertial moment } \\
\text { of the rotator }\end{array}$ & $N \cdot m \cdot s$ \\
\hline$f_{r}$ & $\begin{array}{l}\text { Friction coefficient (due to } \\
\text { the bearing lubrication condition) }\end{array}$ & $N / r p m$ \\
\hline$f_{p}$ & $\begin{array}{l}\text { Friction coefficient (due to } \\
\text { aerodynamics) }\end{array}$ & $N / r p m$ \\
\hline$i_{a}$ & Armature current & $A$ \\
\hline$V_{m}$ & Normalized rotational speed & $r p m$ \\
\hline$u$ & Input voltage & $V$ \\
\hline$T_{0}$ & Non-load torque & $N \cdot m$ \\
\hline$T_{2}$ & Load torque & \\
\hline
\end{tabular}

\section{B. Takagi-Sugeno formulation}

By following the methodology to represent nonlinear systems in Takagi-Sugeno form by using the nonlinear sector approach [13], [14], the nonlinear system given in (1) can be expressed as:

$$
\begin{aligned}
{\left[\begin{array}{c}
\dot{x}_{1}(t) \\
\dot{x}_{2}(t)
\end{array}\right]=} & {\left[\begin{array}{cc}
-\frac{R_{a}}{L} & -\frac{K_{e}}{L} \\
\frac{K_{T}}{J_{1}} & -\left(\frac{f_{r}+\frac{f_{p}}{J_{1}}}{x_{2}} x_{2}(t)\right)
\end{array}\right]\left[\begin{array}{l}
x_{1}(t) \\
x_{2}(t)
\end{array}\right]+} \\
& {\left[\begin{array}{c}
\frac{1}{L} \\
0
\end{array}\right] u(t)+\left[\begin{array}{cc}
0 & 0 \\
-\frac{1}{J_{1}} & -\frac{1}{J_{1}}
\end{array}\right]\left[\begin{array}{c}
T_{0}(t) \\
T_{2}
\end{array}\right] }
\end{aligned}
$$

By considering $x_{2}(t)=\rho(t)$ as the bounded scheduling variable, the system (2) can be written as:

$$
\begin{aligned}
\dot{x}(t) & =A(\rho(t)) x(t)+B u(t)+G \theta(t) \\
y(t) & =C x(t)
\end{aligned}
$$

Using the nonlinear sector approach, we know that $\rho(t)$ varies in a bounded region $\rho(t)=\left[\begin{array}{ll}\rho & \bar{\rho}\end{array}\right]$, where $\rho$ and $\bar{\rho}$ are the lower and upper bounds of $\rho(t)$, so that, system (2) can be rewritten as:

$$
\begin{aligned}
& \dot{x}(t)=\sum_{i=1}^{m} \xi_{i}(\rho(t)) A_{i} x(t)+B u(t)+G \theta(t) \\
& y(t)=C x(t)
\end{aligned}
$$

where

$$
\begin{gathered}
A_{1}=\left[\begin{array}{cc}
-\frac{R_{a}}{L} & -\frac{K_{e}}{L} \\
\frac{K_{T}}{J_{1}} & -\left(\frac{f_{r}+f_{p}}{J_{1}} \bar{\rho}\right)
\end{array}\right], B=\left[\begin{array}{c}
\frac{1}{L} \\
0
\end{array}\right] \\
A_{2}=\left[\begin{array}{cc}
-\frac{R_{a}}{L} & -\frac{K_{e}}{L} \\
\frac{K_{T}}{J_{1}} & -\left(\frac{f_{r}+f_{p}}{J_{1}} \underline{\rho}\right)
\end{array}\right], C=\left[\begin{array}{ll}
0 & 1
\end{array}\right]
\end{gathered}
$$

$$
\theta(t)=\left[\begin{array}{c}
T_{0}(t) \\
T_{2}
\end{array}\right] \text { and } G=\left[\begin{array}{cc}
0 & 0 \\
-\frac{1}{J_{1}} & -\frac{1}{J_{1}}
\end{array}\right]
$$

$m$ is the number of local models (in this case $m=2$ ), $\rho(t)$ is the scheduling variable, $u(t) \in \mathbb{R}^{l}$ is the input, $x(t) \in \mathbb{R}^{n}$ is the state vector, $y(t) \in \mathbb{R}^{p}$ represents the measured output, $\theta(t) \in \mathbb{R}^{s}$ is the unknown input vector, containing the non-load torque $T_{0}(t)$ which in normal operation conditions must be constant. A change in its value implies that a change in the friction torque coefficient has occurred and in consequence a possible bearing fault.

$\xi_{i}(\rho(t))$ are the membership functions given as:

$$
\begin{aligned}
& \xi_{1}(\rho(t))=\eta_{0}^{1}=\frac{\bar{\rho}-\rho(t)}{\bar{\rho}-\underline{\rho}} \\
& \xi_{2}(\rho(t))=\eta_{1}^{1}=1-\eta_{0}^{1}
\end{aligned}
$$

\section{OBSERVER DESIGN}

Now, let us consider the following full-order generalized PI observer for system (4)

$$
\begin{aligned}
\dot{\zeta}(t) & =\sum_{i=1}^{m} \xi_{i}(\rho(t))\left(N_{i}(\zeta(t)+T G \hat{\theta}(t))+H_{i} v(t)+F_{i} y(t)\right)+ \\
J u(t)+T G \hat{\theta}(t) & \\
\dot{v}(t) & =\sum_{i=1}^{m} \xi_{i}(\rho(t))\left(S_{i}(\zeta(t)+T G \hat{\theta}(t))+L_{i} v(t)+M_{i} y(t)\right) \\
\hat{x}(t) & =\zeta(t)+T G \hat{\theta}(t)+Q y(t) \\
\dot{\hat{\theta}}(t) & =\Phi(C \hat{x}(t)-y(t))
\end{aligned}
$$

where $\zeta(t) \in \mathbb{R}^{n}$ represents the state vector of the observer, $v(t) \in \mathbb{R}^{q_{1}}$ is an auxiliary vector, $\hat{x}(t) \in \mathbb{R}^{n}$ is the estimate of $x(t)$ and $\hat{\theta}(t) \in \mathbb{R}^{s}$ is the estimate of $\theta(t)$. Matrices $N_{i}$, $H_{i}, F_{i}, J, S_{i}, L_{i}, M_{i}, T, Q$ and $\Phi$ are unknown matrices of appropriate dimensions which must be determined such that $\hat{x}(t)$ and $\hat{\theta}(t)$ converges asymptotically to $x(t)$ and $\theta(t)$, respectively.

For the sake of simplicity the following notation is used:

$$
\Psi(\rho(t))=\Psi(\rho)=\sum_{i=1}^{m} \xi_{i}(\rho(t)) \Psi_{i}
$$

Thus, the observer (6)-(9) can be rewritten as follows:

$$
\begin{aligned}
\dot{\zeta}(t)= & N(\rho)(\zeta(t)+T G \hat{\theta}(t))+H(\rho) v(t)+F(\rho) y(t)+ \\
& J u(t)+T G \hat{\theta}(t) \\
\dot{v}(t)= & S(\rho)(\zeta(t)+T G \hat{\theta}(t))+L(\rho) v(t)+M(\rho) y(t) \\
\hat{x}(t)= & \zeta(t)+T G \hat{\theta}(t)+Q y(t) \\
\dot{\hat{\theta}}(t)= & \Phi(C \hat{x}(t)-y(t))
\end{aligned}
$$

Now, we introduce the following lemma. 
Lemma 1: There exists an observer of the form (6)-(9) for system (4) if the error dynamics

$$
\dot{\beta}(t)=\mathscr{A}(\rho) \beta(t)
$$

with

$$
\mathscr{A}(\rho)=\left[\begin{array}{ccc}
N(\rho) & H(\rho) & N(\rho) T G+T G \\
S(\rho) & L(\rho) & S(\rho) T G \\
\Phi C & 0 & \Phi C T G
\end{array}\right]
$$

and

$$
\beta(t)=\left[\begin{array}{c}
\varepsilon(t) \\
v(t) \\
\tilde{\theta}(t)
\end{array}\right]
$$

and if there exists a matrix $T$ of appropriate dimension such that the following conditions are satisfied
(a) $N(\rho) T+F(\rho) C-T A(\rho)=0$
(b) $J=T B$
(c) $S(\rho) T+M(\rho) C=0$
(d) $T+Q C=I_{n}$

Proof. Let $T \in \mathbb{R}^{n \times n}$ be a parameter matrix and define the error $\varepsilon(t)=\zeta(t)-T x(t)+T G \theta(t)$, then its derivative is given by

$$
\begin{aligned}
\dot{\varepsilon}(t)= & N(\rho) \varepsilon(t)+H(\rho) v(t)+(N(\rho) T G+T G) \tilde{\theta}(t) \\
& (J-T B) u(t)+(N(\rho) T+F(\rho) C-T A(\rho)) x(t)
\end{aligned}
$$

where $\dot{\theta}(t)=0$ and $\tilde{\theta}(t)=\hat{\theta}(t)-\theta(t)$.

By using the definition of $\varepsilon(t)$ equations (7) and (8) can be rewritten as:

$$
\begin{aligned}
\dot{v}(t)= & S(\rho) \varepsilon(t)+L(\rho) v(t)+(S(\rho) T+M(\rho) C) x(t)+ \\
& S(\rho) T G \tilde{\theta}(t) \\
\hat{x}(t)= & \varepsilon(t)+(T+Q C) x(t)+T G \tilde{\theta}(t)
\end{aligned}
$$

Now, if conditions (a)-(d) of Lemma 1 are satisfied, equations (15)-(17) are simplified as:

$$
\begin{aligned}
\dot{\varepsilon}(t) & =N(\rho) \varepsilon(t)+H(\rho) v(t)+(N(\rho) T G+T G) \tilde{\theta}(t) \\
\dot{v}(t) & =S(\rho) \varepsilon(t)+L(\rho) v(t)+S(\rho) T G \tilde{\theta}(t) \\
e(t) & =\varepsilon(t)+T G \tilde{\theta}(t)
\end{aligned}
$$

where $e(t)=\hat{x}(t)-x(t)$.

Using (20), $\dot{\tilde{\theta}}(t)$ can be written as:

$$
\begin{aligned}
\dot{\tilde{\theta}}(t) & =\dot{\hat{\theta}}(t) \\
& =\Phi C \varepsilon+\Phi C T G \tilde{\theta}(t)
\end{aligned}
$$

Thus, the observer error dynamics is obtained from (18), (19) and (21) as

$$
\dot{\beta}(t)=\mathscr{A}(\rho) \beta(t)
$$

where

$$
\mathscr{A}(\rho)=\left[\begin{array}{ccc}
N(\rho) & H(\rho) & N(\rho) T G+T G \\
S(\rho) & L(\rho) & S(\rho) T G \\
\Phi C & 0 & \Phi C T G
\end{array}\right]
$$

and

$$
\beta(t)=\left[\begin{array}{c}
\varepsilon(t) \\
v(t) \\
\tilde{\theta}(t)
\end{array}\right]
$$

In this case if $\dot{\beta}(t)=\mathscr{A}(\rho) \beta(t)$ is asymptotically stable, then $\lim _{t \rightarrow \infty} e(t)=0$.

Now, the problem of the generalized PI observer design is reduced to find matrices $N(\rho), H(\rho), F(\rho), J, S(\rho)$, $L(\rho), M(\rho), T, Q$ and $\Phi$, such that $\dot{\beta}(t)=\mathscr{A}(\rho) \beta(t)$ is asymptotically stable.

\section{A. Parameterization of the observer matrices}

In this section, the parameterization of the all solutions to the algebraic constraints (a)-(d) is introduced.

Conditions (d) of Lemma 1 can be written as

$$
\left[\begin{array}{ll}
T & Q
\end{array}\right] \Sigma=I_{n}
$$

where $\Sigma=\left[\begin{array}{l}I \\ C\end{array}\right]$. The particular solution to (23) is given by

$$
\left[\begin{array}{ll}
T & Q
\end{array}\right]=\Sigma^{+}
$$

or equivalently

$$
\begin{aligned}
& T=\Sigma^{+}\left[\begin{array}{c}
I_{n} \\
0
\end{array}\right] \\
& Q=\Sigma^{+}\left[\begin{array}{c}
0 \\
I_{p}
\end{array}\right]
\end{aligned}
$$

where $\Sigma^{+}$is any generalized inverse of $\Sigma$, such that it verifies $\Sigma \Sigma^{+} \Sigma=\Sigma$.

From condition (a) and (d) of Lemma 1 we obtain

$$
N(\rho)=K(\rho) C+T A(\rho)
$$

where $K(\rho)=N(\rho) Q-F(\rho)$.

On the other hand, from condition (c) of Lemma 1 we get

$$
S(\rho)=Z(\rho) C
$$

where $Z(\rho)=S(\rho) Q-M(\rho)$.

Remark 1: From the above results, we can see that the determination of the generalized PI observer (6)-(9) can be done as follows: Matrix $F(\rho)$ can be deduced as $F(\rho)=N(\rho) Q-K(\rho)$, matrix $M(\rho)=S(\rho) Q-Z(\rho)$, and matrix $J=T B$. Matrices $Q, N(\rho)$ and $S(\rho)$ are defined in (26), (27) and (28) respectively. On the other hand, parameter matrices $K(\rho), H(\rho), Z(\rho)$ and $L(\rho)$ can be obtained form the stability of (22). 
By using equations (27) and (28) the observer error dynamics (22) can be written as

$$
\left[\begin{array}{c}
\dot{\varphi}(t) \\
\dot{\tilde{\theta}}(t)
\end{array}\right]=\underbrace{\left[\begin{array}{cc}
\mathbb{A}_{1}(\rho)+\mathbb{Y}(\rho) \mathbb{A}_{2} & \mathbb{B}_{1}(\rho)+\mathbb{Y}(\rho) \mathbb{B}_{2} \\
\mathbb{C} & \mathbb{D}
\end{array}\right]}_{\mathscr{A}(\rho)} \underbrace{\left[\begin{array}{c}
\varphi(t) \\
\tilde{\theta}(t)
\end{array}\right]}_{\beta(t)}
$$

where

$$
\begin{gathered}
\varphi(t)=\left[\begin{array}{l}
\varepsilon(t) \\
v(t)
\end{array}\right], \mathbb{A}_{1}(\rho)=\left[\begin{array}{cc}
T A(\rho) & 0 \\
0 & 0
\end{array}\right], \\
\mathbb{A}_{2}=\left[\begin{array}{ll}
C & 0 \\
0 & I
\end{array}\right], \mathbb{B}_{1}(\rho)=\left[\begin{array}{c}
T A(\rho) T G+T G \\
0
\end{array}\right], \\
\mathbb{B}_{2}=\left[\begin{array}{c}
C T G \\
0
\end{array}\right], \mathbb{C}=\left[\begin{array}{ll}
\Phi C & 0
\end{array}\right], \\
\mathbb{D}=\Phi C T G, \quad \mathbb{Y}(\rho)=\left[\begin{array}{cc}
K(\rho) & H(\rho) \\
Z(\rho) & L(\rho)
\end{array}\right] .
\end{gathered}
$$

\section{B. Stability of the proposed observer}

In this section, a method for design a generalized PI Observer is presented.

The following theorem gives the LMIs conditions which allow the determination of all generalized PI Observer matrices.

Theorem 1: There exists a parameter matrix $\mathbb{Y}(\rho)$ such that system (29) is asymptotically stable if and only if there exist a matrix $X=\left[\begin{array}{cc}X_{1} & 0 \\ 0 & X_{2}\end{array}\right]>0$ such that the following LMIs are satisfied

$$
\begin{array}{ll}
{\left[\begin{array}{ll}
\mathbb{A}_{2} & \mathbb{B}_{2}
\end{array}\right]^{T \perp}\left[\begin{array}{rr}
X_{1} \mathbb{A}_{1}(\rho)+\mathbb{A}_{1}^{T}(\rho) X_{1} & X_{1} \mathbb{B}_{1}(\rho)+\mathbb{C}^{T} X_{2} \\
(*) & X_{2} \mathbb{D}+\mathbb{D}^{T} X_{2}
\end{array}\right] \times} \\
{\left[\begin{array}{ll}
\mathbb{A}_{2} & \mathbb{B}_{2}
\end{array}\right]^{T \perp T}<0}
\end{array}
$$

and

$$
\mathbb{D}^{T} X_{2}+X_{2} \mathbb{D}<0
$$

Then, by using the elimination lemma [15] matrix $\mathbb{Y}(\rho)$ is parameterized as

$$
\mathbb{Y}(\rho)=X^{-1}\left(\mathcal{B}_{r}^{+} \mathcal{K}(\rho) \mathcal{C}_{l}^{+}+\mathcal{Z}-\mathcal{B}_{r}^{+} \mathcal{B}_{r} \mathcal{Z} \mathcal{C}_{l} \mathcal{C}_{l}^{+}\right)
$$

where

$$
\begin{aligned}
\mathcal{K}(\rho)= & -\mathcal{R}^{-1} \mathcal{B}_{l}^{T} \vartheta(\rho) \mathcal{C}_{r}^{T}\left(\mathcal{C}_{r} \vartheta(\rho) \mathcal{C}_{r}^{T}\right)^{-1}+ \\
& \mathcal{S}(\rho)^{1 / 2} \mathcal{L}\left(\mathcal{C}_{r} \vartheta(\rho) \mathcal{C}_{r}^{T}\right)^{-1 / 2} \\
\vartheta(\rho)= & \left(\mathcal{B}_{l} \mathcal{R}^{-1} \mathcal{B}_{l}^{T}-\mathcal{D}(\rho)\right)>0 \\
\mathcal{S}(\rho)= & \mathcal{R}^{-1}-\mathcal{R}^{-1} \mathcal{B}_{l}^{T} \vartheta(\rho)- \\
& \left.\vartheta(\rho) \mathcal{C}_{r}^{T}\left(\mathcal{C}_{r} \vartheta(\rho) \mathcal{C}_{r}^{T}\right)^{-1} \mathcal{C}_{r} \vartheta\right] \mathcal{B}_{l} \mathcal{R}^{-1}
\end{aligned}
$$

with

$$
\begin{gathered}
\mathcal{D}(\rho)=\left[\begin{array}{cc}
X_{1} \mathbb{A}_{1}(\rho)+\mathbb{A}_{1}^{T}(\rho) X_{1} & X_{1} \mathbb{B}_{1}(\rho)+\mathbb{C}^{T} X_{2} \\
(*) & X_{2} \mathbb{D}+\mathbb{D}^{T} X_{2}
\end{array}\right], \\
\mathcal{B}=\left[\begin{array}{l}
I \\
0
\end{array}\right] \text { and } \mathcal{C}=\left[\begin{array}{ll}
\mathbb{A}_{2} & \mathbb{B}_{2}
\end{array}\right] .
\end{gathered}
$$

Matrix $\mathcal{Z}$ is arbitrarily chosen, matrix $\mathcal{L}$ must satisfy $\|\mathcal{L}\|<1$ and matrix $\mathcal{R}>0$ must provides matrix $\vartheta(\rho)>0$. Matrices $\mathcal{C}_{l}, \mathcal{C}_{r}, \mathcal{B}_{l}$ and $\mathcal{B}_{r}$ are full rank matrices such that $\mathcal{C}=\mathcal{C}_{l} \mathcal{C}_{r}$ and $\mathcal{B}=\mathcal{B}_{l} \mathcal{B}_{r}$.

Proof. Consider the following Lyapunov function

$$
V(\beta(t))=\beta(t)^{T} X \beta(t)>0
$$

with $X=\left[\begin{array}{cc}X_{1} & 0 \\ 0 & X_{2}\end{array}\right]>0$. Its derivative along the trajectory of (29) is given by

$$
\dot{V}(\beta(t))=\beta(t)^{T}\left(\mathscr{A}^{T}(\rho) X+X \mathscr{A}(\rho)\right) \beta(t)
$$

the inequality $\dot{V}(\beta(t))<0$ is valid for all $\beta(t) \neq 0$ if and only if

$$
\left[\begin{array}{c|c}
\left(\mathbb{A}_{1}(\rho)+\mathbb{Y}(\rho) \mathbb{A}_{2}\right)^{T} X_{1}+ & X_{1}\left(\mathbb{B}_{1}(\rho)+\mathbb{Y}(\rho) \mathbb{B}_{2}\right)+ \\
X_{1}\left(\mathbb{A}_{1}(\rho)+\mathbb{Y}(\rho) \mathbb{A}_{2}\right) & \mathbb{C}^{T} X_{2} \\
\hline(*) & X_{2} \mathbb{D}+\mathbb{D}^{T} X_{2}
\end{array}\right]<0
$$

which can be written as

$$
\mathcal{B X}(\rho) \mathcal{C}+(\mathcal{B X}(\rho) \mathcal{C})^{T}+\mathcal{D}(\rho)<0
$$

where $\mathcal{X}(\rho)=X_{1} \mathbb{Y}(\rho), \mathcal{B}, \mathcal{C}$ and $\mathcal{D}(\rho)$ are defined before. By using the elimination lemma, inequality (39) is equivalent to

$$
\begin{array}{r}
\mathcal{C}^{T \perp} \mathcal{D}(\rho) \mathcal{C}^{T \perp T}<0 \\
\mathcal{B}^{\perp} \mathcal{D}(\rho) \mathcal{B}^{\perp T}<0
\end{array}
$$

with $\mathcal{C}^{T \perp}=\left[\begin{array}{ll}\mathbb{A}_{2} & \mathbb{B}_{2}\end{array}\right]^{T \perp}$ and $\mathcal{B}^{\perp}=\left[\begin{array}{ll}0 & I\end{array}\right]$.

Using the definition of matrices $\mathcal{C}^{T \perp}$ and $\mathcal{D}(\rho)$ the inequality (40) is equivalent to (30), and by using matrices $\mathcal{B}^{\perp}$ and $\mathcal{D}(\rho)$ the inequality (41) is equivalent to (31).

From the elimination lemma, if conditions (40) and (41) are satisfied, the parameter matrix $\mathbb{Y}(\rho)$ is obtained as in (32)-(35).

\section{SIMULATION RESULTS}

Considering the following values for the parameters of the DC motor: $R_{a}=0.60 \Omega, L=0.012 H$, $K_{e}=0.001 \mathrm{~V} / \mathrm{rpm}, K_{T}=0.3 \mathrm{~N} \cdot \mathrm{m} / \mathrm{A}, J_{1}=0.20 \mathrm{~N} \cdot \mathrm{m} \cdot \mathrm{s}$, $f_{r}=0.35 \mathrm{~N} \cdot \mathrm{m} / \mathrm{rpm}, f_{p}=0.0007 \mathrm{~N} \cdot \mathrm{m} / \mathrm{rpm}^{2}$ and $V_{m}(t)=\rho(t)$ varying from $100 \mathrm{rpm}$ to $120 \mathrm{rpm}$, we obtain the following matrices for the Takagi-Sugeno system (4)

$$
\begin{gathered}
A_{1}=\left[\begin{array}{cc}
-50.000 & -0.083 \\
1.500 & -2.134
\end{array}\right], A_{2}=\left[\begin{array}{cc}
-50.000 & -0.083 \\
1.500 & -2.155
\end{array}\right] \\
B=\left[\begin{array}{c}
83.333 \\
0
\end{array}\right], C=\left[\begin{array}{ll}
0 & 1
\end{array}\right] \text { and } G=\left[\begin{array}{cc}
0 & 0 \\
-5 & -5
\end{array}\right]
\end{gathered}
$$

Now, we can obtain the generalized PI observer by considering $X_{2}=I$ in the Lyapunov function (36) to avoid a bilinearity in inequality (31). 
Solving (30) and (31), and considering matrices $\mathcal{R}=\left[\begin{array}{llll}1 & 0 & 0 & 0 \\ 0 & 1 & 0 & 0 \\ 0 & 0 & 1 & 0 \\ 0 & 0 & 0 & 1\end{array}\right] \times 10^{-6}, \mathcal{L}=\left[\begin{array}{lll}0.01 & 0.01 & 0.01 \\ 0.01 & 0.01 & 0.01 \\ 0.01 & 0.01 & 0.01 \\ 0.01 & 0.01 & 0.01\end{array}\right]$ and $\mathcal{Z}^{2}=0$ to solve (32)-(35) we obtain the following observer matrices:

$$
\begin{gathered}
N_{1}=\left[\begin{array}{cc}
-50.000 & 0.274 \\
0.750 & -2.230
\end{array}\right], N_{2}=\left[\begin{array}{cc}
-50.000 & 0.184 \\
0.750 & -2.221
\end{array}\right] \\
H_{1}=\left[\begin{array}{cc}
298.547 & 298.547 \\
43.138 & 43.138
\end{array}\right], H_{2}=\left[\begin{array}{cc}
298.546 & 298.546 \\
43.050 & 43.050
\end{array}\right] \\
F_{1}=\left[\begin{array}{c}
-0.2203 \\
0.0481
\end{array}\right], F_{2}=\left[\begin{array}{c}
-0.2203 \\
0.0330
\end{array}\right], J=\left[\begin{array}{ll}
83.333 & 0 \\
83.333 & 0
\end{array}\right] \\
M_{1}=\left[\begin{array}{cc}
-0.3869 \\
-0.3869
\end{array}\right], M_{2}=\left[\begin{array}{ll}
-0.3869 \\
-0.3869
\end{array}\right] \\
S_{1}=\left[\begin{array}{cc}
0 & 0.7738 \\
0 & 0.7738
\end{array}\right], S_{2}=\left[\begin{array}{ll}
0 & 0.7738 \\
0 & 0.7738
\end{array}\right] \\
L_{1}=L_{2}=\left[\begin{array}{cc}
-1.3112 & 0.0132 \\
0.0132 & -1.3112
\end{array}\right] \times 10^{5}, Q=\left[\begin{array}{c}
0 \\
0.5
\end{array}\right] \\
T=\left[\begin{array}{cc}
1 & 0 \\
0 & 0.5
\end{array}\right] \text { and } \Phi=\left[\begin{array}{l}
22.1137 \\
22.1137
\end{array}\right] .
\end{gathered}
$$

To evaluate the performance of our approach, a simulation was made. In this simulation, a healthy value of $T_{0}$ is fixed as a constant in $0.01 \mathrm{~N} \cdot \mathrm{m}$. If a deviation from this value is detected, then a possible fault has occurred. In a real situation (considering that the operation conditions have not been changed) if this deviation of $T_{0}$ from the nominal value is permanent, then we can conclude that the bearings are no more healthy and they need to be subject of maintenance or to be replaced. To simulate a suddenly fault in the bearing, an abrupt change in $T_{0}$ is made, changing from $0.01 \mathrm{~N} \cdot \mathrm{m}$ to $3 N \cdot m$ (see Fig. 4). $T_{2}$ is considered constant and equal to $0.01 \mathrm{~N} \cdot \mathrm{m}$. Then, the value of $T_{0}$ restored to the nominal value, to verify that the observer follows adequately the profile of $T_{0}$.

Fig. 1 illustrates the simulation scheme. The generalized PI observer is used for fault detection and estimation on bearings by monitoring $T_{0}$.

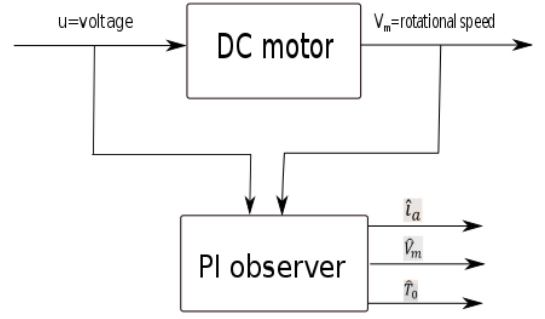

Fig. 1: Simulation scheme of the fault detection.

The initial conditions of the simulation were $\hat{x}(t)=$ $\left[\begin{array}{ll}166.40 & 120\end{array}\right]^{T}$ and $x(t)=\left[\begin{array}{ll}170 & 140\end{array}\right]^{T}$. The estimation of the states are shown in Figs. 1 and 2. It can be seen in these figures that the observer performs well when estimating the states.

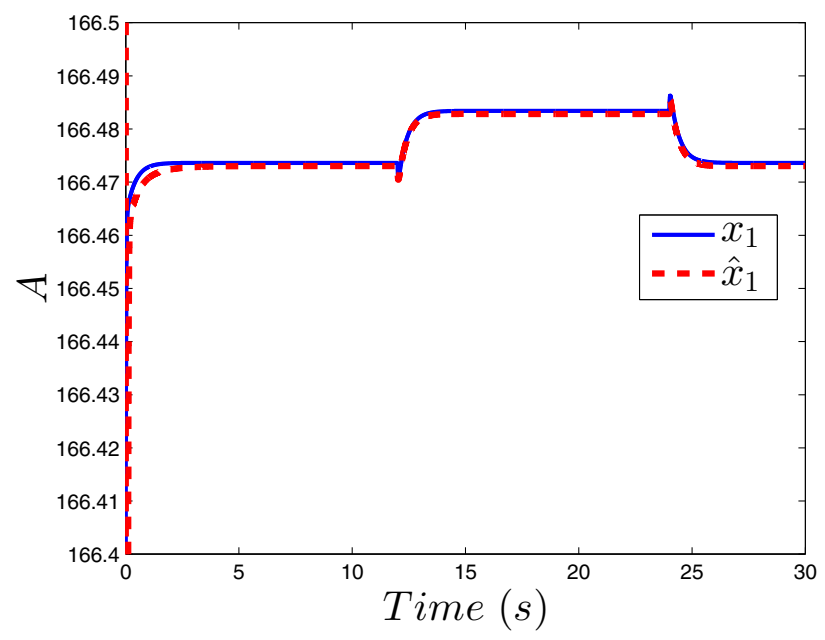

Fig. 2: The estimation of $x_{1}$

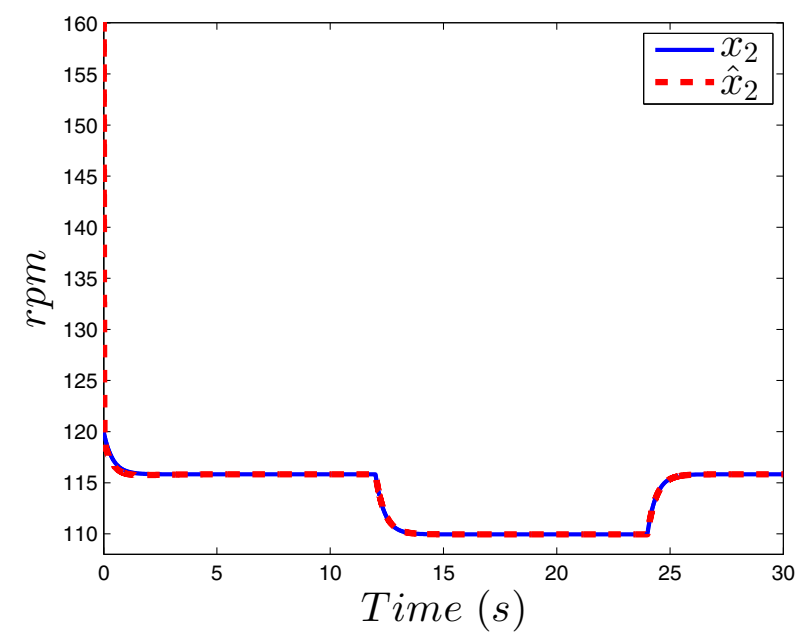

Fig. 3: The estimation of $x_{2}$

The estimation of $T_{0}$ is shown in Fig. 4. Although the observer does not estimate the exact value of $T_{0}$, it is capable 
to detect adequately when this variable changes its value and consequently, that a bearing fault is present in the system.

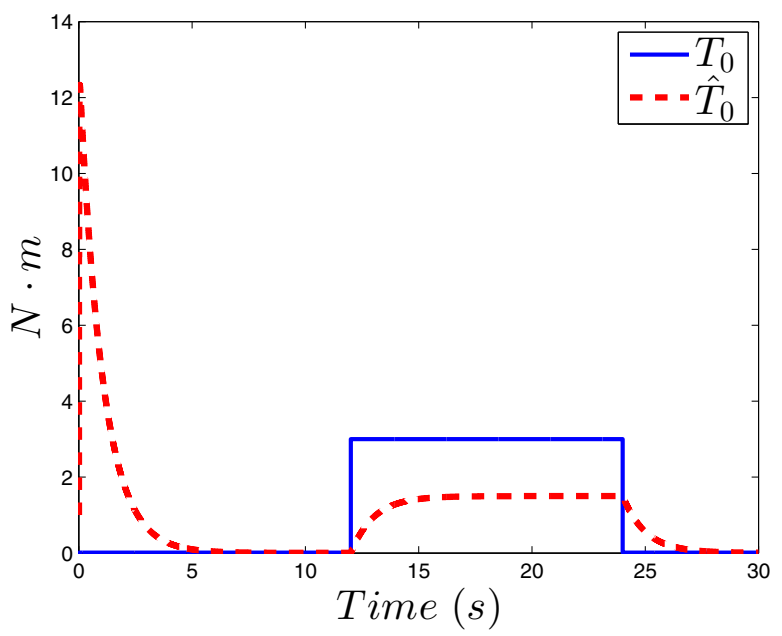

Fig. 4: The estimation of $T_{0}$

\section{CONCLUSIONS}

An observer-based fault detection scheme for supervising the health condition on bearings coupled to DC motors is presented in this work. The Takagi-Sugeno approach is used to obtain a suitable mathematical model of the DC motor to design a proposed generalized proportional-integral observer. This observer is used to monitor the bearing health by estimating on-line the temporal profile of the load torque $T_{0}$. By considering that all time, the DC motor performs its task at the same load operation conditions, a change on $T_{0}$ can be mainly caused by friction or other associated problems on bearings. Simulations of abrupt changes on $T_{0}$ are performed in order to evaluate the observer capabilities to detect these changes and to investigate the feasibility of our approach for future experimental works and other rotative machines.

\section{REFERENCES}

[1] X.-Q. Liu and H.-Y. Zhang, "Fault detection and diagnosis of permanent-magnet DC motor based on parameter estimation and neural network," IEEE Transactions on Industrial Electronics, vol. 47, no. 5, pp. 1021-1030, 2000.

[2] H. Zhang and J. Chen, "Identification of stochastic multi-variable continuous- time systems," International Journal of Modeling Simulation, vol. 10, no. 2, pp. 71-74, 1990.

[3] F. Dominik and R. Isermann., "Hierarchical motor diagnosis utilizing structural knowledge and a self-learning neuro-fuzzy scheme," IEEE Transactions on Industrial Electronics, vol. 47, no. 5, 2000.

[4] L. Menini, C. Possieri, and A. Tornambé, "Appplication of algebraic geometric techniques in permament-magnet DC motor fault detection and identification," European Journal of Control, vol. 25, pp. 39-50, 2015.

[5] L. Menini and A. Tornambe, "On the use of algebraic geometry for thedesign of high-gain observers for continuous-time polynomial systems," in IFAC World Congress, vol. 19, 2014, pp. 43-48.

[6] G. L. Osorio-Gordillo, M. Darouach, L. Boutat-Baddas, and C. M. Astorga-Zaragoza, "Generalized dynamic observers for LPV singular systems," in IFAC-PapersOnLine, vol. 48-26, 2015, pp. 152-157.

[7] G. L. Osorio-Gordillo, M. Darouach, and C. M. Astorga-Zaragoza, " $H_{\infty}$ dynamical observer design for linear descriptor systems," in American Control Conference, Portland, USA, 2014.
[8] G. L. Osorio-Gordillo, M. Darouach, L. Boutat-Baddas, and C. M. Astorga-Zaragoza, " $H_{\infty}$ dynamical observer-based control for descriptor systems," in European Control Conference, Strasbourg, France, 2014.

[9] N. Gao, M. Darouach, M. Alma, and H. Voos, " $H_{\infty}$ descentralized dynamic-observer-based control design for large-scale nonlinear interconnected systems," Transactions on IoT and Cloud Computing, vol. 2, no. 3, pp. 47-69, 2014.

[10] Z. Wang, L. Li, Y. Zhang, and Y. Shen, "Systems observer design for linear descriptor systems," in Chinese Control Conference, 2014.

[11] G. C. Goodwin and R. H. Middleton, "The class of all stable unbiased state estimators," Systems \& Control Letters, vol. 13, pp. 161-163, 1989.

[12] H. J. Marquez, "A frequency domain approach to state estimation," Journal of the Franklin Institute, vol. 340, pp. 147-157, 2003.

[13] H. Othake, K. Tanaka, and H. O. Wang., "Fuzzy modeling via sector nonlinearity concept," Integrated computer-aided engieneering, vol. 10 , no. 4, pp. 333-341, 2003.

[14] Z. Lendek, T. M. Guerra, R. Babuska, and D. S. Bart, Stability analysis and nonlinear obsever design usign Takagi-Sugeno fuzzy models, S.V. B. Heidelberg, Ed., 2010.

[15] R. E. Skelton, T. Iwasaki, and K. Grigoriadis, A unified algebraic approach to linear control design. Taylor \& Francis, 1998. 